\title{
Applications of order-clustered fixed point theorems to generalized saddle point problems and preordered variational inequalities
}

Linsen Xie ${ }^{1}$, Jinlu $\mathrm{Li}^{2^{*}}$ and Wenshan Yang ${ }^{3}$

"Correspondence: jli@shawnee.edu

2 Department of Mathematics, Shawnee State University,

Portsmouth, Ohio 45662, USA Full list of author information is available at the end of the article

\begin{abstract}
In this paper, we introduce the concepts of preordered Banach spaces, generalized saddle points and preordered variational inequalities. Then we apply the order-clustered fixed point theorems to prove the existence of solutions to these problems.

MSC: 06A06; 47B48; 47B60; 49J40

Keywords: chain-complete preordered sets; order-clustered fixed point; Abian-Brown fixed point theorem; order-increasing upward mapping; generalized saddle point; generalized Nash equilibrium; preordered variational inequality
\end{abstract}

\section{Introduction}

In an ordinary game, the sets of strategies of the players or the decision makers may be equipped with a preorder, and the decision makers may consider having indifference (same) utilities at order equivalent elements. It is the motivation to investigate the properties of order equivalent elements and order equivalent classes in a preordered set, which are called order-clusters in [1].

Fixed point theory has played important roles in traditional equilibrium theory, variational inequalities and optimization theory (see [2-12]). Fixed point theorems have been applied in the proofs of the existence of solutions in equilibrium problems, variational inequalities, and other problems, in which the underlying spaces are topological spaces and the considered mappings must satisfy some continuity conditions. One is to extend these techniques from traditional variational analysis to analysis on ordered sets, in which there are order structures which may not be topological structures. Hence some new fixed point theorems on ordered sets must be developed.

For single-valued mappings, Tarski et al. provided a fixed point theorem on complete lattices and on chain-complete posets (see [1]). For set-valued mappings, Fujimoto in [13] generalized Tarski's fixed point theorem from single-valued mappings to set-valued mappings on complete lattices. Very recently, in [6, 7], Li provided several versions of extensions of both the Abian-Brown fixed point theorem and the Fujimoto-Tarski fixed point theorem for set-valued mappings on chain-complete posets. These fixed point theorems 
have been applied to solve vector-complementarity problems and generalized Nash equilibrium problems.

To generalize the fixed point theorems from posets to preordered sets, Xie et al. in [1] introduced the concepts of order cluster and order-clustered fixed point for order-cluster preserving mappings. They also provided some order-clustered fixed point theorems on preordered sets, which have been applied to solve some generalized equilibrium problems for some strategic games with preordered preferences. We recall the fixed point theorem obtained in [1].

Let $(P, \succcurlyeq)$ be a chain-complete preordered set and let $F: P \rightarrow 2^{P} \backslash\{\varnothing\}$ be a set-valued mapping. Suppose that $F$ satisfies the following two conditions:

A1. $F$ is order-increasing upward.

A3. There is an element $y$ in $P$ with $y \preccurlyeq u$, for some $u \in F(y)$.

Assume, in addition to conditions $\mathrm{A} 1$ and $\mathrm{A} 3, F$ also satisfies one of the following conditions:

A2. $\{z \in P: z \preccurlyeq u$ for some $u \in F(x)\}$ is an inductive subset of $P$, for each $x \in P$.

$\mathrm{A} 2^{\prime} . F(x)$ is inductive with a finite number of maximal element clusters, for every $x \in P$.

A2". $F(x)$ has a maximum element, for every $x \in P$.

Then $F$ has an order-clustered fixed point, that is, there are $x^{*} \in P$ and $w \in\left[x^{*}\right]$ with $w \in F\left(x^{*}\right)$.

In this paper, we apply the above fixed point theorem to solve some generalized variational inequalities, in which the ranges of the considered mappings and the underlying spaces both are preordered sets. We also solve generalized saddle point problems and generalized equilibrium problems in preordered sets.

\section{Order-clustered fixed point theorems on preordered sets}

In this section, we recall and provide some concepts and properties of preordered sets and the concept of order-clustered fixed point of set-valued mapping on preordered sets, which is introduced in [1] by Xie et al. Here we closely follow the notations from [2, 7, 14-16] and [1].

Let $P$ be a nonempty set. An ordering relation $\succcurlyeq$ on $P$ is said to be a preorder, whenever it satisfies the following two conditions:

1. (reflexive) $x \succcurlyeq x$, for every $x \in P$.

2. (transitive) $x \succcurlyeq y$ and $y \succcurlyeq z$ imply $x \succcurlyeq z$, for all $x, y, z \in P$.

Then $P$, together with the preorder $\succcurlyeq$, is called a preordered set, which is denoted by $(P, \succcurlyeq)$. Furthermore, a preorder $\succcurlyeq$ on a preordered set $P$ is said to be a partial order if, in addition to the above two properties, it also satisfies

3. (antisymmetric) $x \succcurlyeq y$ and $y \succcurlyeq x$ imply $x=y$, for every $x, y \in P$.

In this case, $(P, \succcurlyeq)$ is simply called a poset.

Remark 2.1 It is worthy to mention for clarification that a preordered set $(P, \succcurlyeq)$ equipped with the preorder $\succcurlyeq$ on $P$ is called a partially ordered system (p.o.s.) in [15].

Let $(P, \succcurlyeq)$ be a preordered set and let $A$ be a nonempty subset of $P$. Then an element $u$ of $P$ is called an upper bound of $A$ if $x \preccurlyeq u$ for each $x \in A$. The collection of all upper bounds 
of $A$ is $\operatorname{denoted}$ by upp $A$. That is,

$$
\operatorname{upp} A=\{y \in P: y \succcurlyeq x \text {, for all } x \in A\} \text {. }
$$

If an upper bound $u$ of $A$ is in $A$, then $u$ is called a greatest element (or a maximum element) of $A$. The collection of all greatest elements (maximum elements) of $A$ is denoted by max $A$, that is,

$$
\max A=\{y \in A: y \succcurlyeq x, \text { for all } x \in A\}
$$

A lower bound of $A$ can be similarly defined and the collection of all lower bounds of $A$ is written as

$$
\text { low } A=\{y \in P: y \preccurlyeq x \text {, for all } x \in A\} \text {. }
$$

The collection of all smallest elements (minimum elements) of $A$ is denoted by $\min A$. That is,

$$
\min A=\{y \in A: y \preccurlyeq x, \text { for all } x \in A\}
$$

If the set of all upper bounds of $A$ has a smallest element, then we call it a supremum of $A$; and the collection of all supremum elements of $A$ is denoted by $\sup A$ or $\vee A$. That is,

$$
\sup A=\vee A=\min (\operatorname{upp} A) .
$$

An infimum of $A$ is similarly defined as a greatest element of the set of all lower bounds of $A$, provided that it exists; and the collection of all infimum elements of $A$ is denoted by $\inf A$ or $\wedge A$. We have

$$
\inf A=\wedge A=\max (\operatorname{low} A) .
$$

It is important to note that, from the above definitions, if $A$ is a subset of a preordered set $(P, \succcurlyeq)$, then $\max A$ and $\min A$ are subsets of $A$; and $\operatorname{upp} A, \operatorname{low} A, \sup A$, and $\inf A$ all are subsets of $P$. In particular, if $(P, \succcurlyeq)$ is a poset, then $\max A, \min A, \sup A$, and $\inf A$ all are singletons, provided that they are nonempty. In this case, $\max A, \min A, \sup A$, and $\inf A$ are simply written by the contained elements, respectively.

An element $y \in A$ is said to be a maximal element of a subset $A$ of a preordered set $(P, \succcurlyeq)$ if for any $z \in A$ with $y \preccurlyeq z$ implies $z \preccurlyeq y$. The collection of all maximal elements of $A$ is denoted by $\operatorname{Max} A$, that is,

$$
\operatorname{Max} A=\{y \in A: x \in A \text { and } x \succcurlyeq y \text { imply } y \succcurlyeq x\}
$$

Similar to the definition of maximal element, a minimal element of $A$ can be defined. The collection of all minimal elements of $A$ is written as

$$
\operatorname{Min} A=\{y \in A: x \in A \text { and } x \preccurlyeq y \text { imply } y \preccurlyeq x\}
$$


Then every greatest element (or smallest element) of a subset $A$ of a preordered set $(P, \succcurlyeq)$ is a maximal element (or minimum element) of $A$; the converse does not hold. That is,

$\max A \subseteq \operatorname{Max} A$ and $\min A \subseteq \operatorname{Min} A, \quad$ for every $\varnothing \neq A \subseteq P$.

A subset $C$ of a preordered set $(P, \succcurlyeq)$ is said to be totally ordered, or a chain in $P$, whenever, for every pair $x, y \in C$, either $x \preccurlyeq y$ or $y \preccurlyeq x$. Following [13] and [10], we have the following.

Definition 2.2 A nonempty subset $A$ of a preordered set $(P, \succcurlyeq)$ is said to be

i. inductive if every chain $C$ in $A$ possesses an upper bound in $A$, that is, $\varnothing \neq$ upp $C \subseteq A$;

ii. chain-complete whenever, every chain $C$ in $A$ has a supremum element in $A$; that is, $\varnothing \neq$ upp $C \subseteq A$.

Let $(P, \succcurlyeq)$ be a preordered set. For any $z, w \in P$, we denote the following $\succcurlyeq$-intervals:

$$
\begin{aligned}
& {[z)=\{x \in P: x \succcurlyeq z\}, \quad(w]=\{x \in P: x \preccurlyeq w\} \quad \text { and }} \\
& {[z, w]=[z) \cap(w]=\{x \in P: z \preccurlyeq x \preccurlyeq w\} .}
\end{aligned}
$$

Let $(P, \succcurlyeq)$ be a preordered set. For any $x, y \in P$, we say that $x, y$ are $\succcurlyeq$-order equivalent ( $\succcurlyeq$-order indifference), which is denoted by $x \sim y$, whenever both $x \succcurlyeq y$ and $y \succcurlyeq x$ hold. It is clear that $\sim$ is an equivalence relation on $P$. For any $x \in P$, let $[x]$ denote the order equivalent class (order indifference class) containing $x$, which is called $\mathrm{a} \succcurlyeq$-cluster (or simply an order cluster, or a cluster, if there is no confusion caused). Let $P / \sim$ or $\tilde{P}$ denote the collection of all order clusters in the preordered set $(P, \succcurlyeq)$. So $x \in[x] \in \tilde{P}$, for every $x \in P$.

In the case that $z \sim w$, we have $[z, w]=[z]$, which is the order cluster containing $z$. It is worthy to note that both $[z)$ and $(w]$ can be considered as unions of order clusters.

Given two preordered sets $\left(X, \succcurlyeq^{X}\right)$ and $\left(U, \succcurlyeq^{U}\right)$, we say that a single valued mapping $f: X \rightarrow U$ is order-increasing (or order-preserving) if $x \preccurlyeq^{X} y$ in $X$ implies $f(x) \preccurlyeq^{U} f(y)$ in $U$. An order-increasing single valued mapping $f: X \rightarrow U$ is said to be strictly orderincreasing whenever $x \prec^{X} y$ implies $f(x) \prec^{U} f(x)$, where $\prec^{X}$ and $\prec^{U}$ are the strict parts of $\succcurlyeq^{X}$ and $\succcurlyeq^{U}$, respectively.

We say that a set-valued mapping $F: X \rightarrow 2^{U} \backslash\{\varnothing\}$ is order-increasing upward if $x \preccurlyeq^{X} y$ in $X$ and $u \in F(x)$ imply that there is $w \in F(y)$ such that $u \preccurlyeq^{u} w . F$ is said to be orderincreasing downward if $x \preccurlyeq^{X} y$ in $X$ and $w \in F(y)$ imply that there is $u \in F(x)$ such that $u \preccurlyeq^{U} w$. A set-valued mapping $F$ is said to be order-increasing whenever $F$ is both orderincreasing upward and downward.

Order-increasing mappings from preordered set to preordered set have the following equivalent classes preserving properties (see [1]).

Lemma 2.3 Let $\left(X, \succcurlyeq^{X}\right)$ and $\left(U, \succcurlyeq^{U}\right)$ be two preordered sets and let $f: X \rightarrow U$ be an orderincreasing single valued mapping. Then, for every $x$ in $X, y \in[x]$ implies $f(y) \in[f(x)]$ in $U$.

Definition 2.4 Let $\left(X, \succcurlyeq^{X}\right)$ and $\left(U, \succcurlyeq^{U}\right)$ be two preordered sets and let $f: X \rightarrow U$ be a single valued mapping. $f$ is said to be order-cluster-preserving, whenever

$$
x \sim^{X} y \text { implies } f(x) \sim^{U} f(y), \quad \text { for } x, y \in X .
$$


Lemma 2.5 Every order-preserving single valued mapping from a preordered set to another preordered set is cluster-preserving.

Definition 2.6 Let $\left(X, \succcurlyeq^{X}\right)$ be a preordered set and let $F: X \rightarrow 2^{X} \backslash\{\varnothing\}$ be a set-valued mapping. An element $x \in X$ is called

1. a fixed point of $F$, whenever $x \in F(x)$;

2. an $\succcurlyeq^{X}$-clustered fixed point (an order-clustered-fixed point, or simply a clustered fixed point) of $F$, whenever there is a $w \in[x]$ such that $w \in F(x)$.

Let $(P, \succcurlyeq)$ be a chain-complete preordered set and let $F: P \rightarrow 2^{P} \backslash\{\varnothing\}$ be a set-valued mapping. Following Fujimoto [13], we use the notation

$$
S F(x)=\{z \in P: z \preccurlyeq u \text { for some } u \in F(x)\}, \quad \text { for every } x \in P .
$$

Xie $e t$ al. in [1] proved the following results, which are extensions of the theorem proved by $\mathrm{Li}$ in [7] from chain-complete posets to chain-complete preordered sets.

Theorem 2.7 Let $(P, \succcurlyeq)$ be a chain-complete preordered set and let $F: P \rightarrow 2^{P} \backslash\{\varnothing\}$ be a set-valued mapping. Suppose that $F$ satisfies the following two conditions:

A1. F is order-increasing upward.

A3. There is an element $y$ in $P$ with $y \preccurlyeq u$, for some $u \in F(y)$.

Assume, in addition to conditions $\mathrm{A} 1$ and $\mathrm{A} 3, F$ also satisfies one of the following conditions:

A2. $S F(x)$ is an inductive subset of $P$, for each $x \in P$.

$\mathrm{A}^{\prime} . F(x)$ is inductive with a finite number of maximal element clusters, for every $x \in P$.

A2". $F(x)$ has a maximum element, for every $x \in P$.

Then $F$ has an order-clustered fixed point, that is, there are $x^{*} \in P$ and $w \in\left[x^{*}\right]$ with $w \in F\left(x^{*}\right)$.

Remark 2.8 If $(P, \succcurlyeq)$ is a chain-complete preordered set satisfying $\wedge P \neq \varnothing$, then condition A3 in Theorem 2.7 will be automatically satisfied.

Proof In the case that $\wedge P \neq \varnothing$, then for any $y \in \wedge P$, we must have $y \preccurlyeq u$, for every $u \in F(y)$.

\section{Generalized saddle point problems for bifunctions on preordered sets}

Let $\left(X, \succcurlyeq^{X}\right)$ and $\left(Y, \succcurlyeq^{Y}\right)$ be preordered sets. The product set $X \times Y$ of the preordered sets $X$ and $Y$ is ordered by the component-wise ordering, which is denoted by $\succcurlyeq^{X \times Y}$. That is, for any $\left(x_{1}, y_{1}\right)$ and $\left(x_{2}, y_{2}\right) \in X \times Y$

$$
\left(x_{1}, y_{1}\right) \succcurlyeq^{X \times Y}\left(x_{2}, y_{2}\right) \text { if and only if } x_{1} \succcurlyeq^{X} x_{2} \text { and } y_{1} \succcurlyeq^{Y} y_{2} \text {. }
$$

One can check that the coordinate ordering $\succcurlyeq^{X \times Y}$ on $X \times Y$ induced by the preorders $\succcurlyeq^{X}$ and $\succcurlyeq^{Y}$ defines a preordering relation on $X \times Y$; and hence $\left(X \times Y, \succcurlyeq^{X \times Y}\right)$ is a preordered set. It has the following properties: 
1. If both $\left(X, \succcurlyeq^{X}\right)$ and $\left(Y, \succcurlyeq^{Y}\right)$ are chain-complete (inductive) preordered sets, then $\left(X \times Y, \succcurlyeq^{X \times Y}\right)$ is also a chain-complete (inductive) preordered set.

2. For any $x \in X$ and $y \in Y,[(x, y)]=[x] \times[y]$, here $[(x, y)],[x],[y]$ are understood to be the order clusters in $\left(X \times Y, \succcurlyeq^{X \times Y}\right),\left(X, \succcurlyeq^{X}\right)$ and $\left(Y, \succcurlyeq^{Y}\right)$, respectively.

3. For any $(x, y),(s, t) \in X \times Y$ with $(x, y) \preccurlyeq^{Y}(s, t)$, then we have

$$
((x, y)]=(x] \times(y], \quad[(x, y))=[x) \times[y) \quad \text { and } \quad[(x, y),(s, t)]=[x, s] \times[y, t]
$$

here, $((x, y)],(x],(y]$ are the order intervals in $\left(X \times Y, \succcurlyeq^{X \times Y}\right),\left(X, \succcurlyeq^{X}\right)$ and $\left(Y, \succcurlyeq^{Y}\right)$, respectively.

Definition 3.1 Let $\left(X, \succcurlyeq^{X}\right),\left(Y, \succcurlyeq^{Y}\right)$ and $\left(U ; \succcurlyeq^{U}\right)$ be preordered sets. Let $C$, $D$ be nonempty subsets of $X$ and $Y$, respectively. Let $F: C \times D \rightarrow U$ be a mapping. A point $\left(x^{*}, y^{*}\right) \in C \times D$ is called a generalized saddle point of the mapping $F$ if it satisfies

$$
F\left(x, y^{*}\right) \preccurlyeq^{U} F\left(x^{*}, y^{*}\right) \preccurlyeq^{U} F\left(x^{*}, y\right), \quad \text { for all } x \in C \text { and } y \in D \text {. }
$$

It is equivalent, for all $x \in C$ and $y \in D$, to

$$
u \preccurlyeq w^{*} \preccurlyeq^{U} v,
$$

for any $u \in\left[F\left(x, y^{*}\right)\right], w^{*} \in\left[F\left(x^{*}, y^{*}\right)\right]$ and $v \in\left[F\left(x^{*}, y\right)\right]$.

Definition 3.2 Let $\left(X, \succcurlyeq^{X}\right),\left(Y, \succcurlyeq^{Y}\right)$ and $\left(U ; \succcurlyeq^{U}\right)$ be preordered sets. Let $C, D$ be nonempty subsets of $X$ and $Y$, respectively. Let $F: C \times D \rightarrow U$ be a mapping. $F$ is said to be

1. order-phase preserving for the first variable on $C$, whenever, for any $x_{2} \succcurlyeq^{X} x_{1}$ in $C$,

$$
F\left(x_{1}, y\right) \preccurlyeq^{U} F\left(x_{1}, t\right) \quad \text { implies } \quad F\left(x_{2}, y\right) \preccurlyeq^{U} F\left(x_{2}, t\right), \quad \text { for any } y, t \in D \text {; }
$$

2. order-phase preserving for the second variable on $D$, whenever, for any $y_{2} \succcurlyeq^{Y} y_{1}$ in $D$,

$$
F\left(x, y_{1}\right) \preccurlyeq^{U} F\left(s, y_{1}\right) \quad \text { implies } \quad F\left(x, y_{2}\right) \preccurlyeq^{U} F\left(s, y_{2}\right), \quad \text { for any } x, s \in C \text {. }
$$

From Definition 2.4, if a mapping $F$ from $\left(C \times D, \succcurlyeq^{X \times Y}\right)$ to $\left(U, \succcurlyeq^{U}\right)$ is order-cluster preserving, then it is order-cluster preserving for every single variable. That is,

1. $s \in[x]$ in $C$ implies $F(s, y) \in[F(x, y)]$ in $U$, for every given $y \in D$;

2. $t \in[y]$ in $D$ implies $F(x, t) \in[F(x, y)]$ in $U$, for every given $x \in C$.

Example 3.3 Let $(R, \geq)$ be the set of real numbers equipped with the ordinary real order $\geq$. Let $F: R \times R \rightarrow R$ be a real function as

$$
F(x, y)=x^{2}-y^{2}, \quad \text { for all } x, y \in R
$$

Then $F$ is order-phase preserving for both variables on $R$.

Theorem 3.4 Let $\left(X, \succcurlyeq^{X}\right),\left(Y, \succcurlyeq^{Y}\right)$ and $\left(U ; \succcurlyeq^{U}\right)$ be preordered sets. Let $C, D$ be nonempty chain-complete subsets of $X$ and $Y$, respectively. Let $F: C \times D \rightarrow U$ be a mapping and satisfy the following conditions: 
1. $F(x, y)$ is order-phase preserving for variables $x$ and $y$ on $C$ and $D$, respectively.

2. For every fixed $x \in C,\{t \in D: F(x, t) \in \min \{F(x, y): y \in D\}\}$ is a nonempty inductive subset of $D$ with a finite number of maximal elements.

3. For every fixed $y \in D,\{s \in C: F(s, y) \in \max \{F(x, y): x \in C\}\}$ is a nonempty inductive subset of $C$ with a finite number of maximal elements.

4. There is an element $\left(x^{\prime}, y^{\prime}\right) \in C \times D$ with $x^{\prime} \preccurlyeq^{X}$ s, for some $s \in C$ satisfying

$$
\begin{gathered}
F\left(s, y^{\prime}\right) \in \max \left\{F\left(x, y^{\prime}\right): x \in C\right\} \\
\text { and } y^{\prime} \preccurlyeq^{Y} t \text {, for some } t \in D \text { satisfying } \\
F\left(x^{\prime}, t\right) \in \min \left\{F\left(x^{\prime}, y\right): y \in D\right\} .
\end{gathered}
$$

5. $F$ is order-cluster preserving on $C \times D$.

Then $F$ has a generalized saddle point.

Proof For the given mapping $F: C \times D \rightarrow U$, we define mappings $\Upsilon_{F}: C \rightarrow 2^{D}$ and $\Psi_{F}$ : $D \rightarrow 2^{C}$ by

$$
\begin{array}{ll}
\Upsilon_{F}(x)=\{t \in D: F(x, t) \in \min \{F(x, y): y \in D\}\}, & \text { for any } x \in C, \\
\Psi_{F}(y)=\{s \in C: F(s, y) \in \max \{F(x, y): x \in C\}\}, & \text { for any } y \in D .
\end{array}
$$

It is worthy to note that since $\Upsilon_{F}(x) \subseteq D$ and $\Psi_{F}(y) \subseteq C$, the above definitions are equivalent to

$$
\begin{array}{ll}
\Upsilon_{F}(x)=\left\{t \in D: F(x, t) \in \bigwedge_{y \in D} F(x, y)\right\}, & \text { for any } x \in C, \\
\Psi_{F}(y)=\left\{s \in C: F(s, y) \in \bigwedge_{x \in C} F(x, y)\right\}, & \text { for any } y \in D .
\end{array}
$$

Define $T: C \times D \rightarrow 2^{C \times D} \backslash\{\varnothing\}$ by

$$
T(x, y)=\Psi_{F}(y) \times \Upsilon_{F}(x), \quad \text { for all }(x, y) \in C \times D .
$$

From conditions 2 and $3, T$ is well defined.

At first, we show that, for any $\left(x_{1}, y_{1}\right),\left(x_{2}, y_{2}\right) \in C \times D,\left(x_{2}, y_{2}\right) \succcurlyeq^{X \times Y}\left(x_{1}, y_{1}\right)$ implies $T\left(x_{1}, y_{1}\right) \subseteq T\left(x_{2}, y_{2}\right)$. For any given $\left(s_{1}, t_{1}\right) \in T\left(x_{1}, y_{1}\right)$, we need to prove $\left(s_{1}, t_{1}\right) \in T\left(x_{2}, y_{2}\right)$. To this end, from that $\left(s_{1}, t_{1}\right) \in T\left(x_{1}, y_{1}\right)$ is equivalent to $s_{1} \in \Psi_{F}\left(y_{1}\right)$ and $t_{1} \in \Upsilon_{F}\left(x_{1}\right)$, we have

$$
F\left(s_{1}, y_{1}\right) \in \max \left\{F\left(x, y_{1}\right): x \in C\right\}
$$

and

$$
F\left(x_{1}, t_{1}\right) \in \min \left\{F\left(x_{1}, y\right): y \in D\right\} .
$$


From (1), we have $F\left(x, y_{1}\right) \preccurlyeq^{U} F\left(s_{1}, y_{1}\right)$, for all $x \in C$. Since $y_{2} \succcurlyeq^{Y} y_{1}$, then from condition 1 in this theorem that $F(x, y)$ is order-phase preserving for variable $y$ on $D$, it implies $F\left(x, y_{2}\right) \preccurlyeq{ }^{U} F\left(s_{1}, y_{2}\right)$, for all $x \in C$. That is, $F\left(s_{1}, y_{2}\right) \in \max \left\{F\left(x, y_{2}\right): x \in C\right\}$; and therefore, $s_{1} \in \Psi_{F}\left(y_{2}\right)$. So we obtain

$$
\Psi_{F}\left(y_{1}\right) \subseteq \Psi_{F}\left(y_{2}\right)
$$

On the other hand, from (2), we have $F\left(x_{1}, t_{1}\right) \preccurlyeq^{U} F\left(x_{1}, y\right)$, for all $y \in D$. Since $x_{2} \succcurlyeq^{X} x_{1}$, then from condition 1 in this theorem that $F(x, y)$ is order-phase preserving for the variable $x$ on $C$, it implies $F\left(x_{2}, t_{1}\right) \preccurlyeq^{U} F\left(x_{2}, y\right)$, for all $y \in D$. That is, $F\left(x_{2}, t_{1}\right) \in \min \left\{F\left(x_{1}, y\right): y \in D\right\}$; and therefore, $t_{1} \in \Upsilon_{F}\left(x_{2}\right)$. So we obtain

$$
\Upsilon_{F}\left(x_{1}\right) \subseteq \Upsilon_{F}\left(x_{2}\right), \quad \text { if } x_{2} \succcurlyeq^{X} x_{1}
$$

Combining (3) and (4), we get

$$
\Psi_{F}\left(y_{1}\right) \times \Upsilon_{F}\left(x_{1}\right) \subseteq \Psi_{F}\left(y_{2}\right) \times \Upsilon_{F}\left(x_{2}\right)
$$

That is, $T\left(x_{1}, y_{1}\right) \subseteq T\left(x_{2}, y_{2}\right)$. It clearly implies that $T$ is order-increasing upward.

We claim that an element $(p, q)$ is a maximal element of $\Psi_{F}(y) \times \Upsilon_{F}(x)$, if and only if $p$ is a maximal element of $\Psi_{F}(y)$ and $q$ is a maximal element of $\Upsilon_{F}(x)$. In fact, if $p$ is a maximal element of $\Psi_{F}(y)$ and $q$ is a maximal element of $\Upsilon_{F}(x)$, then, for any $(s, t) \in \Psi_{F}(y) \times \Upsilon_{F}(x)$, we find that $s \succcurlyeq^{X} p$ implies $p \succcurlyeq^{X} s$ and $t \succcurlyeq^{Y} q$ implies $q \succcurlyeq^{Y} t$. Then we find that $(s, t) \succcurlyeq^{X \times Y}$ $(p, q)$ implies $(p, q) \succcurlyeq^{X \times Y}(s, t)$. So $(p, q)$ is a maximal element of $\Psi_{F}(y) \times \Upsilon_{F}(x)$.

On the other hand, if $p$ is not a maximal element of $\Psi_{F}(y)$ or $q$ is not a maximal element of $\Upsilon_{F}(x)$, say, $p$ is not a maximal element of $\Psi_{F}(y)$, then there is $s \in \Psi_{F}(y)$ with $s \succ^{X} p$, that is, $(s, q) \succ^{X \times Y}(p, q)$. It implies that $(p, q)$ cannot be a maximal element of $\Psi_{F}(y) \times \Upsilon_{F}(x)$. Hence, from conditions 3 and 4 in this theorem, $\left(T(x, y), \succcurlyeq^{X \times Y}\right)$ is an inductive preordered set with a finite number of maximal elements.

Let $\left(x^{\prime}, y^{\prime}\right)$ be the element in $C \times D$ given in condition 4 of this theorem. Then there are points $s \in \Psi_{F}\left(y^{\prime}\right)$ and $t \in \Upsilon_{F}\left(x^{\prime}\right)$ satisfying $x^{\prime} \preccurlyeq^{X} s$, and $y^{\prime} \preccurlyeq^{Y} t$; that is,

$$
(s, t) \in T\left(x^{\prime}, y^{\prime}\right) \text { and } \quad\left(x^{\prime}, y^{\prime}\right) \preccurlyeq^{X \times Y}(s, t) \text {. }
$$

Hence the mapping $T$ from $C \times D$ to $2^{C \times D} \backslash\{\varnothing\}$ satisfies all conditions in Theorem 2.7 with respect to condition $\mathrm{A} 2^{\prime}$. As we apply the mapping $T$ to the preordered set $\left(C \times D, \succcurlyeq^{X \times Y}\right)$, which is a chain complete preordered set, from Theorem 2.7, $T$ has an order-clustered fixed point, say $\left(x^{*}, y^{*}\right) \in C \times D$. Let $\left[\left(x^{*}, y^{*}\right)\right]^{C \times D}$ denote the order-cluster in $\left(C \times D, \succcurlyeq^{X \times Y}\right)$ containing the point $\left(x^{*}, y^{*}\right)$. So there is an element $(u, v) \in\left[\left(x^{*}, y^{*}\right)\right]^{C \times D} \subseteq C \times D$ such that $(u, v) \in T\left(x^{*}, y^{*}\right)$. Then we have

$$
u \in \Psi_{F}\left(y^{*}\right) \text { and } v \in \Upsilon_{F}\left(x^{*}\right) .
$$

It next will be shown that $\Psi_{F}$ and $\Upsilon_{F}$ both are order-cluster preserving on $D$ and $C$, respectively. In fact, from condition $1, F(x, y)$ is order-phase preserving on $C$. 
From $u \in \Psi_{F}\left(y^{*}\right)$, we get $F\left(u, y^{*}\right) \in \max \left\{F\left(x, y^{*}\right): x \in C\right\}$, that is,

$$
F\left(u, y^{*}\right) \succcurlyeq^{U} F\left(x, y^{*}\right), \quad \text { for all } x \in C \text {. }
$$

From condition $5, F$ is order-cluster preserving. From $(u, v) \in\left[\left(x^{*}, y^{*}\right)\right]$, it implies $F\left(u, y^{*}\right) \in$ $\left[F\left(x^{*}, y^{*}\right)\right]$. By $(5)$, we have

$$
F\left(x^{*}, y^{*}\right) \succcurlyeq^{U} F\left(x, y^{*}\right), \quad \text { for all } x \in C .
$$

From $v \in \Upsilon_{F}\left(x^{*}\right)$, we have $F\left(x^{*}, v\right) \in \min \left\{F\left(x^{*}, y\right): y \in D\right\}$, that is,

$$
F\left(x^{*}, v\right) \preccurlyeq^{U} F\left(x^{*}, y\right), \quad \text { for all } y \in D \text {. }
$$

Since $F$ is order-cluster preserving, from $(u, v) \in\left[\left(x^{*}, y^{*}\right)\right]$, it implies $F\left(x^{*}, v\right) \in\left[F\left(x^{*}, y^{*}\right)\right]$. From (7), we have

$$
F\left(x^{*}, y^{*}\right) \preccurlyeq^{U} F\left(x^{*}, y\right), \quad \text { for all } y \in D \text {. }
$$

By combining (6) and (8), we get

$$
F\left(x, y^{*}\right) \preccurlyeq^{U} F\left(x^{*}, y^{*}\right) \preccurlyeq^{U} F\left(x^{*}, y\right), \quad \text { for all } x \in C \text { and } y \in D \text {. }
$$

Hence $\left(x^{*}, y^{*}\right)$ is a generalized saddle point of $F$ on $C \times D$.

If we apply the two mappings $\Upsilon_{F}$ and $\Psi_{F}$ for a mapping $F$, defined in the proof of Theorem 3.4, and apply their order-increasing properties, we can get the following theorem.

Theorem 3.5 Let $\left(X, \succcurlyeq^{X}\right),\left(Y, \succcurlyeq^{Y}\right)$ and $\left(U ; \succcurlyeq^{U}\right)$ be preordered sets. Let $C, D$ be nonempty chain-complete subsets of $X$ and $Y$, respectively. Let $F: C \times D \rightarrow U$ be a mapping and satisfy the following conditions:

1. $\Upsilon_{F}: C \rightarrow 2^{D}$ and $\Psi_{F}: D \rightarrow 2^{C}$ both are order-increasing upward.

2. For every fixed $x \in C,\left(\Upsilon_{F}(x), \succcurlyeq^{Y}\right)$ is a nonempty inductive subset of $D$ with a finite number of maximal elements.

3. For every fixed $y \in D,\left(\Psi_{F}(y), \succcurlyeq^{X}\right)$ is a nonempty inductive subset of $C$ with a finite number of maximal elements.

4. There is an element $\left(x^{\prime}, y^{\prime}\right) \in C \times D$ with $x^{\prime} \preccurlyeq^{X} s$, for some $s \in \Psi_{F}\left(y^{\prime}\right)$ and $y^{\prime} \preccurlyeq^{Y} t$, for some $t \in \Upsilon_{F}\left(x^{\prime}\right)$.

5. F is order-cluster preserving on $C \times D$.

Then $F$ has a generalized saddle point.

Sketch of the proof Let $T: C \times D \rightarrow 2^{C \times D} \backslash\{\varnothing\}$ be defined as in the proof of Theorem 3.4:

$$
T(x, y)=\Psi_{F}(y) \times \Upsilon_{F}(x), \quad \text { for all }(x, y) \in C \times D
$$

For any $\left(x_{1}, y_{1}\right),\left(x_{2}, y_{2}\right) \in C \times D$ with $\left(x_{2}, y_{2}\right) \succcurlyeq^{X \times Y}\left(x_{1}, y_{1}\right)$, take an arbitrary element $\left(s_{1}, t_{1}\right) \in$ $T\left(x_{1}, y_{1}\right)$, we have $s_{1} \in \Psi_{F}\left(y_{1}\right)$ and $t_{1} \in \Upsilon_{F}\left(x_{1}\right)$. Since $y_{2} \succcurlyeq^{Y} y_{1}$ and $x_{2} \succcurlyeq^{X} x_{1}$, from condition 1 in this theorem, there are $s_{2} \in \Psi_{F}\left(y_{2}\right)$ and $t_{2} \in \Upsilon_{F}\left(x_{2}\right)$ such that $s_{2} \succcurlyeq^{X} s_{1}$ and $t_{2} \succcurlyeq^{Y} t_{1}$. 
That is $\left(s_{2}, t_{2}\right) \in C \times D$ satisfying $\left(s_{2}, t_{2}\right) \succcurlyeq^{X \times Y}\left(s_{1}, t_{1}\right)$ and $\left(s_{2}, t_{2}\right) \in T\left(x_{2}, y_{2}\right)$. Hence $T$ is an $\succcurlyeq^{X \times Y}$-increasing mapping from $C \times D$ to $2^{C \times D} \backslash\{\varnothing\}$. The rest of the proof is similar to the proof of Theorem 3.4 .

\section{Generalized equilibrium problems of bifunctions on preordered sets}

Definition 4.1 Let $\left(X, \succcurlyeq^{X}\right)$ and $\left(U ; \succcurlyeq^{U}\right)$ be preordered sets. Let $C$ be a nonempty subset of $X$. Let $F: C \times C \rightarrow U$ be a mapping. A point $x^{*} \in C$ is called a generalized equilibrium of the mapping $F$ if it satisfies

$$
F\left(x^{*}, x\right) \preccurlyeq^{U} F\left(x^{*}, x^{*}\right), \quad \text { for all } x \in C \text {. }
$$

It is equivalent to the following, for all $x \in C$ :

$$
u \preccurlyeq^{u} w^{*},
$$

for any $u \in\left[F\left(x^{*}, x\right)\right]$ and $w^{*} \in\left[F\left(x^{*}, x^{*}\right)\right]$.

For a given mapping $F: C \times C \rightarrow U$, define a mapping $\Gamma: C \rightarrow 2^{C}$ by

$$
\begin{aligned}
\Gamma_{F}(x) & =\left\{t \in C: F(x, t) \in \bigvee_{y \in C} F(x, y)\right\} \\
& =\{t \in C: F(x, t) \in \max \{F(x, y): y \in C\}\}, \quad \text { for any } x \in C .
\end{aligned}
$$

We prove the following theorem for the existence of generalized equilibrium. The proof is similar to the proofs of Theorems 3.4 and 3.5 .

Theorem 4.2 Let $\left(X, \succcurlyeq^{X}\right)$ and $\left(U ; \succcurlyeq^{U}\right)$ be preordered sets. Let $C$ be a nonempty chaincomplete subset of $X$. Let $F: C \times C \rightarrow U$ be a mapping and satisfy the following conditions:

1. $F(\cdot, y)$ is order-phase preserving on $C$, for every $y \in C$.

2. For every fixed $x \in C,\left(\Gamma_{F}(x), \succcurlyeq^{X}\right)$ is a nonempty inductive subset of $C$ with a finite number of maximal elements.

3. There is an element $x^{\prime} \in C$ with $x^{\prime} \preccurlyeq^{X} s$, for some $s \in \Gamma_{F}\left(x^{\prime}\right)$.

4. $F$ is order-cluster preserving on $C \times C$.

Then $F$ has a generalized equilibrium in $C$.

Proof For the given mapping $F$, by condition 2 in this theorem, the mapping $\Gamma_{F}: C \rightarrow$ $2^{C} \backslash\{\varnothing\}$ is well defined. It will next be shown that $\Gamma_{F}$ is order-increasing upward. For any $x_{1}, x_{2} \in C$ with $x_{2} \succcurlyeq^{X} x_{1}$, for arbitrary $t_{1} \in \Gamma_{F}\left(x_{1}\right)$, we have

$$
F\left(x_{1}, t_{1}\right) \in \max \left\{F\left(x_{1}, y\right): y \in C\right\} .
$$

That is, $F\left(x_{1}, y\right) \preccurlyeq^{U} F\left(x_{1}, t_{1}\right)$, for all $y \in D$. Since $x_{2} \succcurlyeq^{X} x_{1}$, then from condition 1 in this theorem we see that $F(x, y)$ is order-phase preserving with respect to $x \in C$, it implies $F\left(x_{2}, y\right) \preccurlyeq^{U} F\left(x_{2}, t_{1}\right)$, for all $y \in C$. That is, $F\left(x_{2}, t_{1}\right) \in \max \left\{F\left(x_{2}, y\right): y \in C\right\}$. We obtain $t_{1} \in \Gamma_{F}\left(x_{2}\right)$. It implies that $\Gamma_{F}$ is order-increasing upward. From condition 3 in this theorem, it is clearly seen that the elements $x^{\prime} \in C, s \in \Gamma_{F}\left(x^{\prime}\right)$ satisfy $x^{\prime} \preccurlyeq s$. Hence the mapping 
$\Gamma_{F}$ from $C$ to $2^{C} \backslash\{\varnothing\}$ satisfies all conditions in Theorem 2.7 with respect to condition $A 2^{\prime}$, as we consider the mapping is from the chain-complete preordered set $\left(C, \succcurlyeq^{X}\right)$ to $2^{C} \backslash\{\varnothing\}$. We denote by $\left[x^{*}\right]_{C}$ the order cluster in $\left(C, \succcurlyeq^{X}\right)$ containing $x^{*}$. So $\Gamma_{F}$ has an order-clustered fixed point, say $x^{*} \in C$. So there is $u \in\left[x^{*}\right]_{C} \subseteq C$, such that $u \in \Gamma_{F}\left(x^{*}\right)$. Then we have

$$
F\left(x^{*}, u\right) \in \max \left\{F\left(x^{*}, y\right): y \in C\right\}
$$

which is equivalent to

$$
F\left(x^{*}, u\right) \succcurlyeq^{u} F\left(x^{*}, y\right), \quad \text { for all } y \in C \text {. }
$$

Since $F$ is order cluster preserving, from $u \in\left[x^{*}\right]_{C}$, it implies $F\left(x^{*}, u\right) \in\left[F\left(x^{*}, x^{*}\right)\right]$. By (9), we have

$$
F\left(x^{*}, x^{*}\right) \succcurlyeq^{U} F\left(x^{*}, y\right), \quad \text { for all } y \in C .
$$

\section{Order-clustered fixed point theorems and generalized preordered-variational inequalities}

In this section, we consider preordered topological spaces and their properties. We also give some fixed point theorems on preordered topological spaces, which will be applied to solving generalized preordered-variational inequalities in preordered Banach spaces. The notations and terminologies used in this section are derived from Aliprantis and Burkinshaw [14], Carl and Heikkilä [2], Li [7], Ok [16], Xie et al. [1].

Let $(P, \succcurlyeq)$ be a preordered set equipped with a topology $\tau$ (it is also a topological space). The topology $\tau$ is called a natural topology on $(P, \succcurlyeq)$ with respect to the preorder $\succcurlyeq$, whenever, for every $z \in P$, the $\succcurlyeq$-intervals $[z)$ and $(z]$ are all $\tau$-closed. A preordered set $(P, \succcurlyeq)$ equipped with a natural topology $\tau$ is called a preordered topological space, and it is denoted by $(P, \tau, \succcurlyeq)$.

A preorder $\succcurlyeq$ on a vector space $P$ is said to be convex, whenever for any $z \in P$, the $\succcurlyeq$-intervals $[z)$ and $(z]$ are all convex subsets in $P$.

We say that a Banach space $(B,\|\cdot\|)$ endowed with a preorder $\succcurlyeq$ is a preordered Banach space if its norm topology is a natural topology with respect to the preorder $\succcurlyeq$ on $B$. A preordered Banach space $(B,\|\cdot\|, \succcurlyeq)$ is called a convex preordered Banach space if the preorder $\succcurlyeq$ is convex on $B$.

Recall that every convex subset in a Banach space is closed in the norm topology if and only if it is closed in the weak topology. Then we have the following result.

Theorem 5.1 Let $(B,\|\cdot\|)$ be a Banach space with norm $\|\cdot\|$. Let $\succcurlyeq$ be a convex preorder on $B$. Then, with respect to the convex preorder $\succcurlyeq$, the norm topology on $B$ is a natural topology, if and only if the weak topology on B is a natural topology.

The connections between the chain-completeness and compactness of subsets in ordered topological spaces are very important topics in the theory of ordered topological spaces. In [6], Li proved that every nonempty compact subset of a partially ordered Hausdorff topological space is chain-complete. In this paper, we extend it to preordered Hausdorff topological spaces. The proof is similar to the proof of Theorem 2.3 in [6] and it is omitted here. 
Theorem 5.2 Every nonempty compact subset of a preordered Hausdorff topological space is chain-complete.

Notice that nonempty bounded closed and convex subset of a reflexive Banach space is compact in the weak topology. From Theorems 5.1 and 5.2, we have the following.

Corollary 5.3 Let $(B, \succcurlyeq)$ be a convex preordered reflexive Banach space. Then, for any nonempty bounded closed and convex subset $P$ of $B,(P, \succcurlyeq)$ is a chain-complete preordered set.

Applying Theorems 2.7, 5.1 and 5.2, we obtain the following result.

Theorem 5.4 Let $(B,\|\cdot\|, \succcurlyeq)$ be a convex preordered reflexive Banach space and let $P$ be a nonempty bounded closed convex subset of B. Let $F: P \rightarrow 2^{P} \backslash\{\varnothing\}$ be a set-valued mapping. Suppose that $F$ satisfies the following two conditions:

A1. F is order-increasing upward.

A3. There is an element $y$ in $P$ with $y \preccurlyeq u$, for some $u \in F(y)$.

Assume, in addition conditions $\mathrm{A} 1$ and $\mathrm{A} 3, F$ also satisfies one of the conditions below:

A2. $S F(x)$ is an inductive subset of $P$, for each $x \in P$.

A2'. $F(x)$ is inductive with a finite number of maximal element clusters, for every $x \in P$.

$\mathrm{A} 2^{\prime \prime} . F(x)$ has a maximum element, for every $x \in P$.

Then $F$ has an order-clustered fixed point, that is, there are $x^{*} \in P$ and $w \in\left[x^{*}\right] \cap P$ with $w \in F\left(x^{*}\right)$.

Let $\left(B, \succcurlyeq^{B}\right)$ and $\left(U ; \succcurlyeq^{U}\right)$ be Banach spaces. As usual, we denote by $L(B, U)$ the collection of all linear mappings from $B$ to $U$.

Definition 5.5 Let $\left(B, \succcurlyeq^{B}\right)$ and $\left(U ; \succcurlyeq^{U}\right)$ be preordered Banach spaces and $C$ a nonempty subset of $B$. Let $F: C \rightarrow L(B, U)$ be a mapping. A generalized preordered-variational inequality problem associated with $C, F$, and $U$, denoted by $\operatorname{GPVI}(F, C, U)$, is to find a point $x^{*} \in C$, such that

$$
F\left(x^{*}\right)\left(x-x^{*}\right) \succcurlyeq^{U} 0, \quad \text { for all } x \in C,
$$

where 0 is understood as the origin of the Banach space $U$. Any such point $x^{*} \in C$ is called a solution to the problem $\operatorname{GPVI}(F, C, U)$.

Notice that $F\left(x^{*}\right) \in L(B, U)$, then (10) is equivalent to

$$
F\left(x^{*}\right)(x) \succcurlyeq^{u} F\left(x^{*}\right)\left(x^{*}\right), \quad \text { for all } x \in C .
$$

Definition 5.6 Let $\left(B, \succcurlyeq^{B}\right)$ and $\left(U ; \succcurlyeq^{U}\right)$ be preordered Banach spaces, and $C$ a nonempty subset of $B$. A mapping $F: C \rightarrow L(B, U)$ is said to be order-phase preserving on $C$, whenever for any $x_{2} \succcurlyeq^{B} x_{1}$ in $C, F\left(x_{1}\right)(y) \preccurlyeq^{U} 0$ implies $F\left(x_{2}\right)(y) \preccurlyeq^{U} 0$, for any given $y \in B$. 
Theorem 5.7 Let $\left(B, \succcurlyeq^{B}\right)$ be a convex preordered reflexive Banach space and $\left(U ; \succcurlyeq^{U}\right) a$ preordered Banach space. Let $C$ be a nonempty bounded closed convex subset of $B$. Let $F: C \rightarrow L(B, U)$ be a mapping and satisfy the following conditions:

1. $F(x): B \rightarrow U$ is preorder-phase preserving on $C$, for every $x \in C$.

2. For every fixed $x \in C$, the set $\left(\{t \in C: F(x)(t) \in \min \{F(x)(y): y \in C\}\}, \succcurlyeq^{B}\right)$ is a nonempty inductive subset of $C$ with a finite number of maximal order clusters.

3. There are elements $x^{\prime}, s \in C$ with $x^{\prime} \preccurlyeq^{B} s$, such that $F\left(x^{\prime}\right)(s) \in \min \left\{F\left(x^{\prime}\right)(y): y \in C\right\}$.

4. $F(x): B \rightarrow U$ is order-cluster preserving, for every $x \in C$.

Then the problem $\operatorname{GPVI}(F, C, U)$ has a solution.

Proof Since $\left(B, \succcurlyeq^{B}\right)$ is a convex preordered reflexive Banach space and $C$ is a nonempty bounded closed convex subset of $B$, from Corollary 5.3, $\left(C, \succcurlyeq^{B}\right)$ is chain-complete. Define a mapping $\Lambda_{F}$ by

$$
\Lambda_{F}(x)=\{t \in C: F(x)(t) \in \min \{F(x)(y): y \in C\}\}, \quad \text { for any } x \in C .
$$

From condition 2 in this theorem, $\Lambda_{F}: C \rightarrow 2^{C} \backslash\{\varnothing\}$ is well defined. Similarly to the proof of Theorem 4.2, we can show that the mapping $\Lambda_{F}$, from $C$ to $2^{C} \backslash\{\varnothing\}$, satisfies all conditions in Theorem 5.4 with respect to condition $\mathrm{A}^{\prime}$. So $\Lambda_{F}$ has an order-clustered fixed point, say $x^{*} \in C$. Then there is $u \in\left[x^{*}\right] \cap C$, such that $u \in \Lambda_{F}\left(x^{*}\right)$. Then we have

$$
F\left(x^{*}\right)(u) \in \min \left\{F\left(x^{*}\right)(y): y \in C\right\},
$$

which is equivalent to

$$
F\left(x^{*}\right)(u) \preccurlyeq^{u} F\left(x^{*}\right)(y), \quad \text { for all } y \in C .
$$

Since $F$ is order-cluster preserving, from $u \in\left[x^{*}\right] \cap C$, it implies $F\left(x^{*}\right)(u) \in\left[F\left(x^{*}\right)\left(x^{*}\right)\right]$. By (12), we have

$$
F\left(x^{*}\right)\left(x^{*}\right) \preccurlyeq^{u} F\left(x^{*}\right)(y), \quad \text { for all } y \in C .
$$

Hence the point $x^{*}$ satisfies (11). It is equivalent to

$$
F\left(x^{*}\right)\left(x-x^{*}\right) \succcurlyeq^{U} 0, \quad \text { for all } x \in C \text {. }
$$

\section{Competing interests}

The authors declare that they have no competing interests.

\section{Authors' contributions}

All authors contributed equally to the writing of this paper. All authors read and approved the final manuscript.

\section{Author details}

${ }^{1}$ Department of Mathematics, Lishui University, Lishui, Zhejiang 323000, China. ${ }^{2}$ Department of Mathematics, Shawnee State University, Portsmouth, Ohio 45662, USA. ${ }^{3}$ Department of Mathematics, Zhejiang Normal University, Jinhua, Zhejiang 321000, China.

\section{Acknowledgements}

The authors sincerely thank the anonymous reviewers for their valuable suggestions, which improved the presentation of this paper. The first author was partially supported by the National Natural Science Foundation of China (11171137).

The third author was partially supported by the National Natural Science Foundation of China (Grant 11101379). 


\section{References}

1. Xie, LS, Li, JL, Yang, WS: Order-clustered fixed point theorems on chain-complete preordered sets and their applications to extended and generalized Nash equilibria. Fixed Point Theory Appl. 2013, 192 (2013)

2. Carl, S, Heikkilä, S: Fixed Point Theory in Ordered Sets and Applications: From Differential and Integral Equations to Game Theory. Springer, New York (2010)

3. Crouzeix, JP: Pseudomonotone variational inequality problems: existence of solutions. Math. Program. 78(3), 305-314 (1997)

4. Isac, G: Complementarity Problems. Lecture Notes in Mathematics, vol. 1528. Springer, Berlin (1992)

5. Isac, G, Li, JL: Complementarity problems, Karamardian's condition and a generalization of Harker-Pang condition. Nonlinear Anal. Forum 6(2), 383-390 (2001)

6. Li, JL: Fixed point theorems on partially ordered topological Vector Spaces and their applications to equilibrium problems with incomplete preferences. J. Fixed Point Theory Appl. (to appear)

7. Li, JL: Several extensions of the Abian-Brown fixed point theorem and their applications to extended and generalized Nash equilibria on chain-complete posets. J. Math. Anal. Appl. 409, 1084-1092 (2014)

8. Li, JL, Ok, EA: Optimal solutions to variational inequalities on Banach lattices. J. Math. Anal. Appl. 388(2), 1157-1165 (2012)

9. Von Neumann, J, Morgenstern, O: The Theory of Games and Economic Behavior. Princeton University Press, Princeton (1944)

10. Xie, LS, Li, JL, Yang, WS: Vector and ordered variational inequalities and applications to order-optimization problems on Banach lattices. J. Appl. Math. 2013, Article ID 439394 (2013)

11. Yao, JC: Variational inequalities with generalized monotone operators. Math. Oper. Res. 19(3), 691-705 (1994)

12. Zhang, CJ: Set-Valued Analysis with Applications in Economics (Chinese). Sciences Press, Beijing (2004)

13. Fujimoto, T: An extension of Tarski's fixed point theorem and its applications to isotone complementarity problems. Math. Program. 28, 116-118 (1984)

14. Aliprantis, CD, Burkinshaw, O: Positive Operators. Springer, Dordrecht (2006)

15. Dunford, N, Schwartz, JT: Linear Operators, Part I. Wiley, New York (1988)

16. Ok, EA: Order theory (forthcoming)

doi:10.1186/1687-1812-2014-191

Cite this article as: Xie et al.: Applications of order-clustered fixed point theorems to generalized saddle point problems and preordered variational inequalities. Fixed Point Theory and Applications 2014 2014:191.

\section{Submit your manuscript to a SpringerOpen ${ }^{\ominus}$ journal and benefit from:}

- Convenient online submission

- Rigorous peer review

- Immediate publication on acceptance

Open access: articles freely available online

- High visibility within the field

- Retaining the copyright to your article 